\title{
Role of VEGF/VEGFR in the pathogenesis of leukemias and as treatment targets (Review)
}

\author{
GUANHUA SONG, YANMEI LI and GUOSHENG JIANG
}

\begin{abstract}
Key Laboratory for Tumor Immunology and Traditional Chinese Medicine Immunology, Key Laboratory for Rare and Uncommon Diseases of Shandong Province, Department of Hemato-Oncology, Institute of Basic Medicine, Shandong Academy of Medical Sciences, Key Laboratory of Ministry of Health for Biotech-Drug, Key Laboratory for Modern Medicine and Technology of Shandong Province, Jinan, Shandong, P.R. China
\end{abstract}

Received June 15, 2012; Accepted August 22, 2012

DOI: 10.3892/or.2012.2045

\begin{abstract}
Angiogenesis plays an important role in solid tumor growth, progression and metastasis. Evidence suggests that the progression of hematolymphoid malignancies also depends on the induction of new blood vessel formation under the influence of acute leukemia, myelodysplastic syndromes, myeloproliferative neoplasms, multiple myeloma and lymphomas. The vascular endothelial growth factor (VEGF) is the most important proangiogenic agent that activates receptors on vascular endothelial cells and promotes blood vessel regeneration. It has been demonstrated that VEGF/VEGF receptor (VEGFR) expression is upregulated in several types of hematolymphoid tumor cells accompanied with angiogenesis. The levels of VEGF/VEGFR are correlated with the treatment, relapse and prognosis of hematolymphoid tumors. In order for VEGF family and their receptors as antiangiogenic targets to treat solid tumors, several antiangiogenic agents targeting VEGF-related pathways have been used for the treatment of hematolymphoid malignancies in clinical trials. The results demonstrate a promising therapeutic intervention in multiple types of hematolymphoid tumors. This review aims to summarize recent advances in understanding the role of VEGF and angiogenesis in leukemias, mainly focusing on their upstream transcriptors, downstream targets and the correlation of VEGF/VEGFR with the treatment, relapse or prognosis of leukemia. The progress of VEGF and its receptors as attractive targets for therapies are also discussed in clinical application.
\end{abstract}

\section{Contents}

1. Introduction

2. Brief review of VEGF/VEGFR

Correspondence to: Dr Guosheng Jiang, Department of HematoOncology, Institute of Basic Medicine, Shandong Academy of Medical Sciences, Jingshi Road 18877, Jinan 250062, Shandong, P.R. China E-mail: jianggsh@hotmail.com

Key words: leukemia, vascular endothelial growth factor, vascular endothelial growth factor receptor, angiogenesis, antiangiogenesis
3. Signaling pathway of VEGF/VEGFR in leukemia

4. In vivo studies of VEGF/VEGFR in patients with leukemia

5. Antiangiogenic therapy in leukemias

6. Conclusion

\section{Introduction}

Angiogenesis is a tightly regulated process dealing with the development of new blood vessels from a pre-existing vascular network. In solid tumor growth, angiogenesis is critical for tumor development, progression and metastasis $(1,2)$. Several types of leukemias similar to solid tumors were also reported to have high in bone marrow microvessel density (MVD) (3). This suggests that angiogenesis plays an important role in the progression of hematolymphoid malignancies. Often, angiogenesis is maintained by a balance of endogenous antiangiogenic and proangiogenic factors. However, the exact mechanism triggering vascular endothelial growth factor (VEGF) expression in hematolymphoid tumors is unknown; different mechanisms similar to those observed in solid tumors are anticipated. In recent years, the expression level of VEGF/VEGF receptor (VEGFR) in patients with different hematolymphoid tumors has been detected and related to reduced survival and lower remission rates (4). Studies have demonstrated that VEGF/VEGFR-related pathways are the most relevant regulators of neoangiogenesis, vasculogenesis and recruitment of endothelial progenitor cells. Furthermore, VEGF/VEGFR interactions may stimulate proliferation, migration and survival of leukemia/lymphoma cells by autocrine and paracrine loops (3). According to a number of studies, acute leukemia cells secrete significant amounts of VEGF in the serum and malignant hematopoietic cells were found to express VEGF and its receptors (5). The clinical outcome of this approach has also been confirmed, and recently coordinated efforts in research have resulted in a number of novel antiangiogenic agents (6). This review focuses on the current knowledge of angiogenesis and antiangiogenic therapies in hematolymphoid malignancies. It mainly demonstrates the role of VEGF/VEGFR in hematological malignancies including its important role in growth, proliferation, survival, as well as the correlation of VEGF/VEGFR with the treatment, relapse or 
prognosis of leukemia. The progress of VEGF and its receptors as attractive targets for therapies are discussed together with their clinical application in leukemic diseases.

\section{Brief review of VEGF/VEGFR}

VEGF, a glycoprotein, was first purified from the in vitro culture medium of bovine pituitary folliculo-stellate cells by Ferrara and Henzel (7). Named after its mitogenic activity for vascular endothelial cells, VEGF was considered a highly specific co-mitogen for the vascular endothelial cells that promoted vascular permeability (8). The five subtype members in the VEGF family are VEGF-A, PIGF, VEGF-B, VEGF-C and VEGF-D. VEGF-A is the prototype of the ligand and is commonly known as VEGF. All these ligands promote endothelium regeneration and increase vascular permeability via binding to three transmembrane receptor tyrosine kinases which are VEGFR-1, -2 and -3. VEGFR-1 ligands include VEGF-A, -B, and placental growth factor (PIGF). VEGFR-2 (also known as KDR in human and Flk-1 in mice) has ligands of VEGF-A, -C, -D and is predominantly expressed in vascular endothelial cells. The activation of VEGFR-2 is necessary and sufficient in order to mediate VEGF-dependent angiogenesis and the induction of vascular permeability $(8,9)$. The binding of VEGFR-3 to the VEGF homologues VEGF-C and VEGF-D is largely restricted to lymphatic endothelial cells and plays an important role in the regulation of lymphangiogenesis $(9,10)$.

Receptor tyrosine kinases are expressed in adult endothelial cells except the brain. VEGFR-1 is also expressed in hematopoietic, monocytes and smooth muscle cells $(11,12)$. VEGFR-2 is expressed mostly in vascular endothelial cells, and also in neuronal, megakaryocytes and hematopoietic stem cells (13). Although the exact contribution of VEGFR-1 signaling to angiogenesis is unclear, it has been shown that VEGFR-1 directly cooperates with VEGFR-2 via heterodimerization similar to the binding of two additional VEGF homologues, VEGF-B and PIGF (12). It has been discovered by Brekken et al (14) that VEGF-2 plays a significant role in VEGF-induced angiogenesis and the binding between VEGF and the VEGFR-2 may activate the mitogen-activated protein kinase (MAPK) system via protein kinase C (PKC) or the Ras protein to induce the proliferation of vascular endothelial cells.

It has been identified that VEGF is the specific growth factor for angiogenesis, while other growth factors including fibroblast growth factor (FGF) and platelet-derived growth factor (PDGF) have no specificity since they act on several different types of cells including vascular endothelial cells. Moreover, VEGF may regulate the development of hemopoietic stem cells and remodel both the extracellular matrix and the regeneration of inflammatory cytokines $(15,16)$. Several leukemic cell strains and primary cells synthesizing and secreting VEGF may affect and modulate the malignant biological behavior of leukemic cells by two positive-feedback loops which are paracrine and autocrine $(17,18)$. VEGF secreted by leukemic cells interacts with relevant receptors on the endothelial cell surface and stimulates endothelial cells to produce growth factors that act on leukemic cells resulting in an increase in their proliferative activity and drug resistance. Hence, antiangiogenesis therapy based on the principle of inhibiting the physiological function of VEGF has become a novel target for antiangiogenic therapy (19).

\section{Signaling pathway of VEGF/VEGFR in leukemia}

Upstream regulators target VEGF/VEGFR expression in leukemic diseases. Much research has focused on the factors regulating VEGF expression and their functions in leukemic diseases from different aspects. VEGF expression is regulated by several intrinsic and extrinsic factors, with hypoxia and hypoglycemia being the major stimuli (20). Often, hypoxiainduced transcription of VEGF mRNA is mediated by the binding of hypoxia-inducible factor-1 (HIF-1) (21); intratumoral hypoxia and HIF-1 mediation have been discovered to be a key angiogenesis triggering event $(22,23)$. This basic helix-loop-helix transcription factor may not only contribute to hypoxia-induced VEGF production, but may also play a critical role in the oncogene-dependent expression of VEGF (24). Additional recent data suggest that HIF-1 $\alpha$ may also be involved in BCR/ABL-dependent expression of VEGF in $\mathrm{Ba} / \mathrm{F} 3$ cells $(25,26)$. HIF- $1 \alpha$ also accounts for the molecular mechanism of autocrine regulation of VEGF in chronic lymphocytic leukemia (CLL) B cells. Ghosh et al (24) reported that CLL B cells express constitutive levels of HIF-1 $\alpha$ under normoxia. The stabilized HIF- $1 \alpha$ may form an active complex with the transcriptional coactivator p300 and phosphorylatedSTAT3 at the VEGF promoter and recruit RNA polymerase II to upregulate VEGF transcription. Consequently, VEGF is secreted at higher levels in CLL B cells. The authors examined the status of the von Hippel-Lindau gene product (pVHL) that dealt with HIF-1 $\alpha$ degradation and discovered it was at a notably lower level in CLL B cells compared with that in normal B cells. This initial evidence explained the aberrant autocrine VEGF secretion in CLL cells. Besides BCR/ABL, microvesicles (MVs) released by malignant cancer cells constituting an important part of the tumor microenvironment may also activate the HIF-1 $\alpha$ pathway with VEGF production in B-cell CLL patients. Ghosh et al (27) demonstrated that MVs circulating in the plasma of B-cell CLL patients exhibited a phenotypic shift from the predominant platelet derived in early stage to leukemic B-cell derived at advanced stage. Furthermore, the total MV level in patients with CLL was higher compared to that of healthy patients. Apart from being a factor in angiogenesis these results indicate that VEGF is also an essential mediator in other clinical phenomena.

As described, VEGF production is associated with the constitutive activity of Janus kinase 3 (Jak3) and the c-Jun $\mathrm{N}$-terminal kinases (JNKs). Jak3 has been suggested to play a key role in the transformation of CTCL T cells since Jak3 inhibitors trigger apoptosis and inhibit cell growth and spontaneous cytokine production of malignant $\mathrm{T}$ cells (28-30). It is proposed that the oncogene Stat3, the primary target of Jak3-mediated transformation, requires coactivators such as HIF-1 $\alpha$ to induce VEGF expression. Additionally, c-Jun phosphorylation and its ability to bind to a VEGF promoter element relating to JNK activity and VEGF production indicate that JNK induced VEGF expression is caused by an increase in c-Jun/AP-1 activity. Activation of the JNK/AP-1 signaling pathway has previously been implicated in the induction of VEGF transcription (31). However, the JNKs have been shown to promote VEGF expression through other mechanisms, such as increasing VEGF mRNA stability (32). Therefore, inhibition of VEGF-inducing pathways or neutralization of VEGF 
itself may imply novel therapeutic modalities in cutaneous T-cell lymphoma (CTCL) (33). In a previous study, researchers discovered that lysophosphatidic acid (LPA) protected CLL cells from apoptosis through a higher expression of LPA receptors and autocrine production of VEGF. Kumar et al (34) reported that an increase in VEGF by LPA was mediated through the activation of JNK and transcription factor $\mathrm{NF}-\kappa \mathrm{B}$ since blocking JNK or NF- $\mathrm{NB}$ activation may inhibit LPA to induce VEGF expression. Furthermore, it was demonstrated that LPA protected cells from apoptosis by blocking the activation of both VEGFR-1 and VEGFR-2 via the VEGF receptor kinase inhibitor. Knocking down the expression of VEGFR-1 and inhibiting the activation of NF- $\kappa$ B and JNK may also block LPA to avoid apoptosis. We hypothesize that LPA contributing to VEGF production in B cell malignancies leads to cell survival (35).

Leukemia is an angiogenesis-dependent malignancy $(36,37)$ and angiogenesis is strictly dependent on Akt/NF- $\mathrm{BB}$ activation. The inhibitors of the $\mathrm{NF}-\kappa \mathrm{B}$ pathway decreasing VEGF secretion in leukemic cells and inhibiting endothelial cell activities may cause the interruption of a reciprocal stimulatory loop between leukemic and endothelial cells. Different reports demonstrating the activation of NF- $\kappa \mathrm{B}$ in lymphoid and myeloid malignancies underscore the implication in malignant transformation (38). While the overexpression of $\mathrm{NF}-\kappa \mathrm{B}$ may lead to chemoresistance, the appropriate inhibition of this pathway may lead to successful therapy. Moreover, the transcription of VEGF by the classical NF- $\kappa \mathrm{B}$ target gene may be repressed, which is one aspect of the participation in tumorigenesis of adult T-cell leukemia (ATL) (39). NF- $\kappa \mathrm{B}$ is also activated by the phosphoinositide 3-kinase (PI3K)/Akt signaling pathway, which is also crucial to several aspects of cell growth, survival and apoptosis. PI3K/Akt activation has been implicated in both the pathogenesis and the progression of a variety of neoplasms which includes leukemias (40). Accumulated evidence over the years has indicated that the $\mathrm{PI} 3 \mathrm{~K} / \mathrm{Akt}$ signal transduction pathway is a major factor for cancer resistance in conventional therapies. Indeed, pharmacologic inhibitors of PI3K/Akt have been found to potentiate the apoptotic action of anti-leukemic drugs (41). Therefore, targeting $\mathrm{NF}-\kappa \mathrm{B}$ activation as well as its upstream regulator Akt may constitute an additional strategy to improve conventional therapies.

Several extensively characterized transcription factors which include activator protein-1 (AP-1), NF- $\kappa \mathrm{B}$ and stimulatory protein-1 (SP-1) modulate VEGF expression. The activation and binding of these transcription factors in tumor cells contribute to VEGF transcription and tumor metastasis (42-44). Among those, AP-1 is a critical factor in the regulation of VEGF gene expression. Pollmann et al (45) determined that VEGF gene expression is mostly regulated by AP-1 and c-Jun in human promyelocytic leukemia (HL-60) cells. Poulaki et al (46) demonstrated that the insulin-like growth factor-1 receptor (IGF-1R) in thyroid tumor cell membranes may promote VEGF production by enhancing the activity of transcription factor AP-1 (46). Rutin in combination with vitamin $\mathrm{E}$ has been demonstrated to synergistically inhibit oxidative damage. Chuang et al (47) reported that rutin in combination with vitamin $\mathrm{E}$ attenuated VEGF expression in HL-60 cells by decreasing the activity of AP-1.
The BTB domain (named after the Drosophila transcription factors Bric-a-brac, Tramtrack and Broad) of promyelocytic leukemia zinc finger (PLZF) as a novel apoptotic and antiangiogenic protein, may directly inhibit tube formation and migration of endothelial cells on Matrigel in vitro. To date, the BTB domain is reported to reduce the expression of VEGF, p-Ak, and p-eNOS in HUVECs. Akt and eNOS play significant roles in angiogenesis stimulated by VEGF which is known to stimulate Akt-dependent phosphorylation of eNOS. These observations reveal that the BTB domain has little or no effect on non-phosphorylated Akt and eNOS. These data indicate that VEGF is essential to the BTB domain function and they form a positive feedback loop to facilitate leukemiarelated disease (48) (Fig. 1).

$\mathrm{BCR} / \mathrm{ABL}$ (an oncogene fusion protein consisting of BCR and ABL, which is associated with the Philadelphia chromosome) functions as a constitutive tyrosine kinase leading to autophosphorylation (49) and activates multiple signaling molecules including p21Ras (50), signal transducer and activator of transcription 5 (STAT5) (51-53) and phosphoinositide 3-kinase (PI3-kinase) (54). Using both single-marker analysis and haplotype analysis, BCL-2 SNP was found to demonstrate consistent association with susceptibility to chronic myeloid leukemia (CML) (55). Recently, it was reported that treatment with Bcr-Abl-targeting siRNAs and imatinib resulted in an enhanced VEGF suppression in K562 cells (56). Little is known about the biochemical mechanisms and signaling pathways contributing to BCR/ABL-induced expression of angiogenic growth factors in CML cells. Böhm et al (57) reported that $\mathrm{BCR} / \mathrm{ABL}$ induces VEGF production in $\mathrm{CML}$ cells through a pathway involving PI3-kinase and mammalian target of rapamycin (mTOR). mTOR has recently been implicated in leukemic cell growth, tumor-associated angiogenesis and the expression of VEGF in acute myeloid leukemia (AML). mTOR-targeting drugs exert anti-leukemic effects on AML cells in vitro through multiple actions, including direct inhibition of proliferation, induction of apoptosis and suppression of VEGF (57).

Downstream target genes of VEGF in leukemias. In addition to stimulating angiogenesis, other studies have demonstrated that VEGF may directly stimulate proliferation of several types of leukemia cells. For example, VEGF stimulates multiple myeloma (MM) cells to migrate, proliferate and survive on fibronectin via autocrine and paracrine loops, which usually contributes to the binding of VEGF and VEGFR-2 (58). As to the modulation of its downstream target gene, a great deal of progress has been made. VEGF induces the expression of heat shock protein 90 (Hsp90), which binds Bcl-2 and Apaf-1 to increase leukemic cell resistance to serum deprivation-induced apoptosis (59). Moreover, VEGF may play an important role in the growth of hematologic neoplasms via a paracrine mechanism. When endothelial cells are exposed to recombinant human VEGF, they may increase mRNA expression of several hematopoietic growth factors, including G-CSF, GM-CSF, stem cell factor (SCF) and IL-6, which act as growth factors for myeloid and lymphoid cells $(60,61)$. In other words, VEGF may promote tumorigenesis by enhancing the production of hematopoietic growth factors. Both VEGF and PKC $\beta$ II were reported to have higher levels of expression 


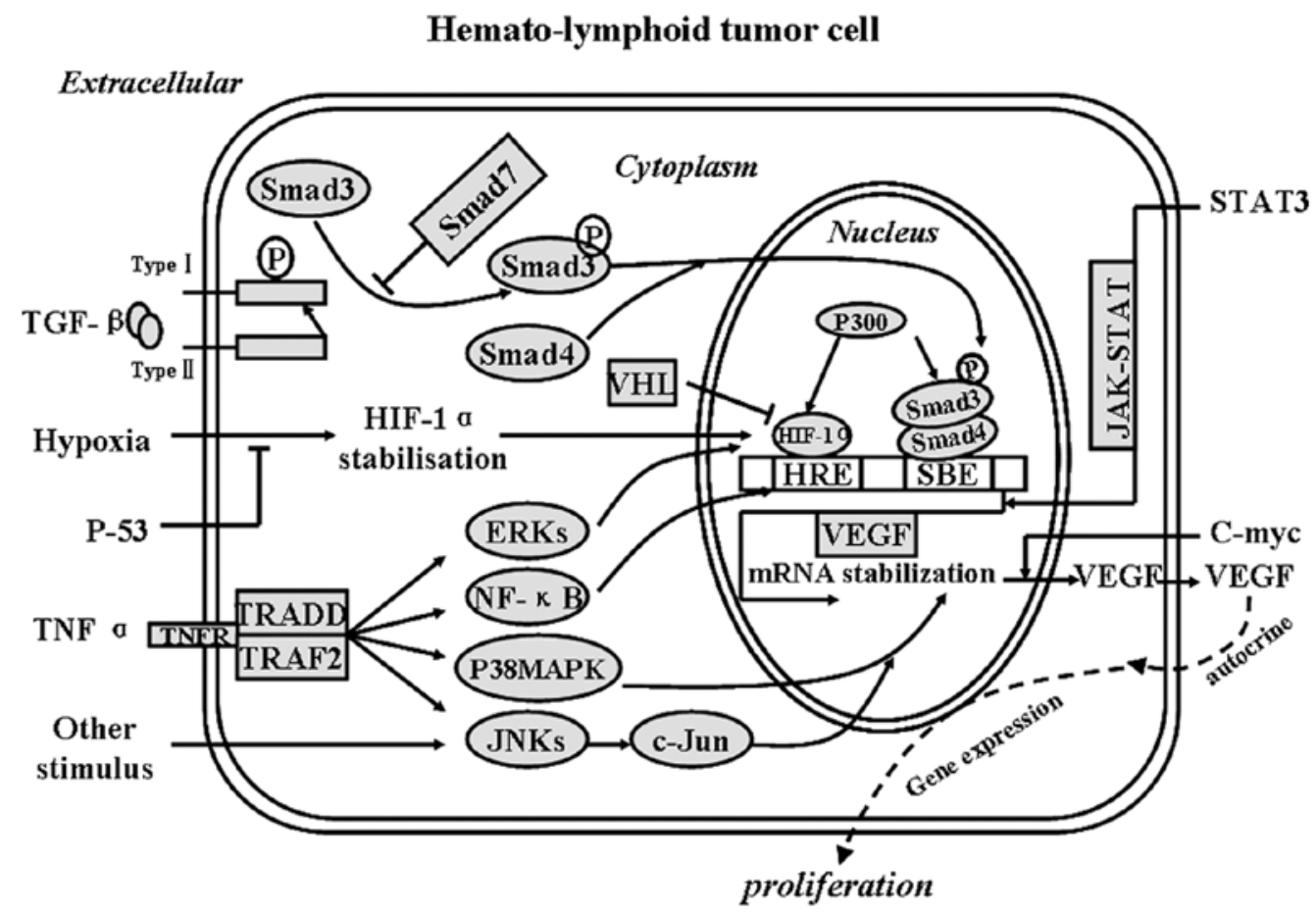

Figure 1. Upstream regulators target VEGF/VEGFR expression in leukemias. Smads, signal transducers and transcription factors in the TGF- $\beta$ signaling pathway; TRADD, protein which binds adaptor protein TRAF2 and then suppresses TRAF2-mediated apoptosis; TRAF2, TNF receptor-associated factor 2, which mediates the signal transduction from members of the TNF receptor superfamily; VHL, von Hippel-Lindau tumor-suppressor also known as pVHL; ERKs, extracellular signal-regulated kinases; P38MAPK, P38 mitogen-activated protein kinases; JNKs, c-Jun N-terminal kinases; p300, a transcriptional coactivator. TGF- $\beta$, hypoxia and TNF- $\alpha$ induce the expression of VEGF and VEGFR in hematolymphoid tumor cells through the Smad family, HIF-1 $\alpha$, ERKs, NF-kB, p38-MAPK and JNKs, respectively, which, ultimately, affects VEGF/VEGFR expression.

related to disease stage and tumor burden $(18,62,63)$. The regulation of PKC $\beta$ expression in CLL cells by VEGF was important since the level of PKC $\beta$ expression in malignant cells in a Tcl1 mouse model determined the development and progression of the disease. Controlling PKC $\beta$ expression is likely to be an important step in CLL pathogenesis. VEGF, due to its role in stimulating PKC $\beta$ expression, is therefore central to the pathogenesis of the disease (64). Migration of B-cell chronic lymphocytic leukemia (B-CLL) cells involves several molecules, including matrix metalloproteinase-9 (MMP-9) and VEGF. Downregulation of MMP-9 by VEGF significantly inhibited the migration of B-CLL cells through human umbilical vein endothelial cells. STAT1 was found to be responsible for MMP-9 downregulation since STAT1 gene silencing restored MMP-9 production and B-CLL cell migration in the presence of VEGF. The VEGF/VEGFR-2 axis is upstream of STAT1 tyrosine phosphorylation thus the inhibition of B-CLL cell migration is ultimately due to the effect of VEGF (65).

VEGF-C has been recognized as a tumor lymphangiogenic factor based on the effects of activated VEGF-R3 on lymphatic endothelial cells. VEGF-C enhances c-Jun binding to the cyclic adenosine 3',5'-monophosphate-response element of the cyclooxygenase-2 (COX-2) promoter and induces COX-2 expression, which may catalyze one of the rate-limiting steps in prostanoid biosynthesis (66) and enhance the survival and proliferation of malignant cells, while negatively influencing anti-tumor immunity. In addition, the VEGF-R3/JNK/AP-1 pathway also participates in the induction of COX-2 by VEGF-C in leukemic cells. VEGF-R2 activation may induce the upregulation of COX-2 via 38 MAPK and JNK signaling pathways in human vascular endothelial cells (67). In line with this study, VEGF-A may also induce the upregulation of COX-2 in leukemic cells. By acting in an autocrine/paracrine manner, VEGF-C may contribute to tumor angiogenesis through the induction of COX-2/prostanoids in subsets of leukemia. A previous study demonstrated that there is a significantly higher induction of VEGF-C by COX-2 in another context (68). Hence, the cross talk between VEGF-C and COX-2 may initiate a positive feedback loop, resulting in enhanced expression of COX-2 and increased synthesis of prostanoids, which lead to a further increase in VEGF-C activity.

The expression of the antiapoptotic myeloid cell leukemia-1 (MCL-1) gene, leading to the enhanced survival of tumor cells, is a novel prognostic factor in B-CLL $(69,70)$. VEGF and interleukin-6 (IL-6) are able to upregulate MCL-1 via autocrine signaling loops. VEGF may be a positive autocrine regulator of MCL-1 in B-CLL. In addition, specific downregulation of MCL-1 gene expression was discovered to promote apoptosis and death of primary B-CLL cells, suggesting the possibility of the inhibition of VEGF, and its pathway may prove useful in the treatment of B-CLL patients (71) (Fig. 2).

\section{In vivo studies of VEGF/VEGFR in patients with leukemia}

$V E G F / V E G F R$ in acute lymphocytic leukemia (ALL). To the best of our knoweldge, Perez-Atayde et al (72) first demonstrated that leukemia progression was correlated to increased bone marrow vascularization. It was demonstrated that ALL patients had an increased blood vessel content compared 


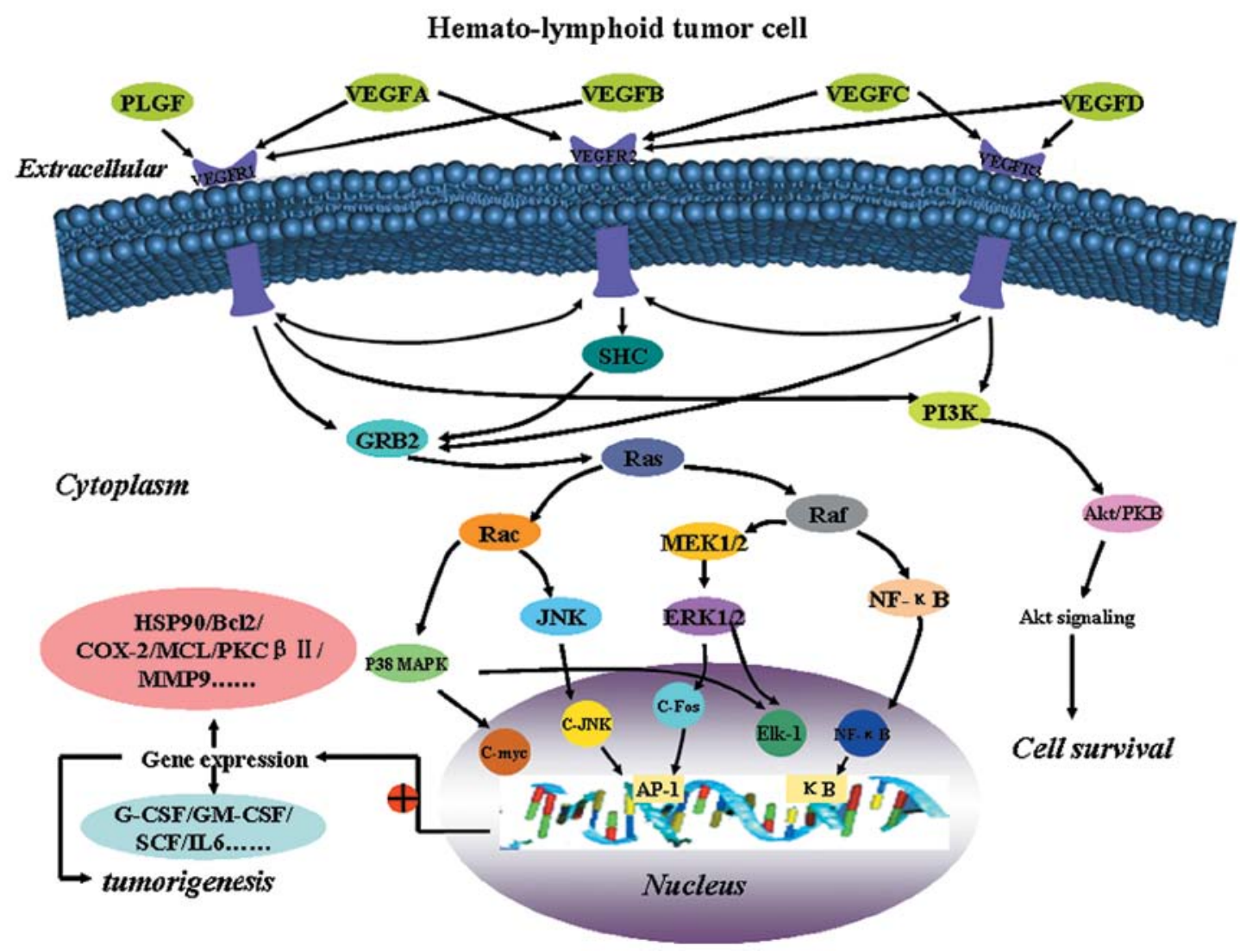

Figure 2. Downstream regulators target VEGF/VEGFR expression in leukemias. After interaction with factors in both the cytoplasm and nucleus, VEGF/VEGFR may effect tumorigenesis by upregulating HSP90, Bcl2, COX-2, MCL, PKC $\beta I I$, MMP9, and downregulating G-CSF, GM-CSF and IL-6.

to their normal counterparts. There were elevated levels of proangiogenic growth factors including basic fibroblast growth factor (bFGF) and VEGF in urine and peripheral blood samples from ALL patients. The factors were correlated with bone marrow angiogenesis $(72,73)$. The existence of an 'angiogenesis switch' first proposed for solid tumors is suggested to apply to hematolymphoid malignancies (3). The 'angiogenesis switch' in leukemias is indicated to increase bone marrow microvessel density (MVD), the expression of HIF-1, multiple proangiogenic factors (VEGF, bFGF and angiopoietin-2) and soluble VEGFR, but a decrease in the expression of endogenous angiogenesis inhibitors (74,75). In a recent study, MVD was found to be higher in T-ALL compared to B-ALL patients (76). In the B-ALL group, cases with $\mathrm{t}(12 ; 21)$ were characterized by a low MVD, while patients with hyperdiploid leukemia displayed a high MVD (3). The correlation between MVD and white blood cell count (WBC) may be a determination of high-risk for B-ALL patients. In addition, patients with a high marrow reticulin fiber density and high MVD exhibit an unfavorable outcome, indicating the possible cellular origin of certain proangiogenic factors (76). The following clinical studies support the capacity of leukemia cells to produce proangiogenic growth factors including VEGF and bFGF in vitro (77).

To further detect whether tumor progression is in concert with the induction of tumor angiogenesis in leukemia, angiogenesis was characterized by immunohistochemical staining of factor VIII (FVIII) in bone marrow biopsies and was quantified by MVD assessment in patients with newly diagnosed ALL or after the completion of remission induction chemotherapy. The results suggested that the leukemia was angiogenesis-dependent. This increased the possibility for antiangiogenic drugs to treat leukemia (78). The high plasma VEGF concentration was linked to leukemic cell invasion in adult T-cell leukemia (ATL) and the major source was from ATL cells themselves (79). Noteworthy, all cell lines were observed to express mRNA and protein of VEGFR-1. In clinical specimens, similar results were also detected in all (100\%) of 11 and $8(73 \%)$ of 11 ATL patients, respectively. VEGF was effectively bound only to VEGFR-1-expressing cells. Further study demonstrated that the key role of VEGF in ATL is to assist in cell invasion, not proliferation. ATL cells upregulate their own chemotaxis to facilitate the invasion into various organs through the combination of VEGF and VEGFR-1 (80). Besides that, the angiogenic effect of VEGF seems to be dependent on nitric oxide (NO). The associations among functional polymorphisms in VEGF $(-2578 \mathrm{C}>\mathrm{A},-1154 \mathrm{G}>\mathrm{A}$ and $-634 \mathrm{G}>\mathrm{C})$ and NOS3 $(-786 \mathrm{~T}>\mathrm{C}$, intron $4 \mathrm{~b}>\mathrm{a}$ and Glu298Asp) were examined with the prognosis of childhood ALL. The results indicate that polymorphisms of VEGF and NOS3 genes are highly associated with the risk of relapse, therefore it may be a prognostic sign in childhood ALL (81).

VEGF/VEGFR in acute myeloid leukemia. In recent studies, the expression of VEGF/VEGFR in AML patients has been detected. The increased levels of plasma VEGF have been correlated with reduced survival and lower remission rates (4), and the level of plasma/serum VEGF was found to be related to the 
number of circulating blasts (82). In addition to the modulation of bone marrow angiogenesis by VEGF from leukemia cells, it was confirmed that the expression of endothelial-specific tyrosine kinase receptors, such as VEGFR-1, -2 and -3 , are also observed in leukemic cells $(83,84)$. De Bont et al $(85)$ reported that new vessel formation was the result of angiogenesis and vasculogenesis. The degree of neovascularization in the bone marrow was correlated with VEGF expression in the leukemic cells. The bone vessel count (MVC) was higher in 23 cases of AML patients compared to that in the normal controls, and the cell proliferation, bone marrow angiogenesis and expression of VEGF were correlated to each other in patients with AML (85). To further assess cellular VEGF levels and their prognostic significance in newly diagnosed AML, a radioimmunoassay (RIA) was performed to quantify VEGF levels in stored samples from 99 patients diagnosed with AML. Although the patients with an increased VEGF level had a shorter survival, there was no evidence to demonstrate the significant relationship between VEGF level and WBC or blast count in AML. In contrast, the results suggest that the cellular VEGF level is an independent predictor of outcome in AML (86). Interestingly, the co-expression of CD147 and VEGF may indicate a poor prognosis in AML and may be a highly sensitive marker for predicting the clinical outcome of patients (87).

As to the induction of angiogenesis by VEGF in AML, Fielder et al (88) investigated the expression of VEGF and its receptors in fresh leukemic blasts and addressed the possible loops for the stimulation of AML blasts. The autocrine VEGF worked through VEGFR-2, by activating eNOS to produce NO through PI3-K/Akt kinase, and maintained clonogenic cell growth in the OCI/AML-2 cell line (89). Also, Fielder et al (90) reported that VEGFR-3 represented a cloned member of class III receptor tyrosine kinase with VEGFR-1 and VEGFR-2. The ligand of VEGFR-3 has been identified as VEGF-C that shares sequence homology with VEGF and PIGF. VEGF-C expression was discovered in leukemic samples of 4 out of 7 VEGFR-3-positive and 4 out of 6 VEGFR-3-negative patients (90). MVD was increased in the bone marrow of patients with AML, supporting the hypothesis that angiogenesis plays an important role in AML (61). High VEGF-C mRNA expression in AML blasts is related to drug resistance in vitro and in vivo. The prognostic significance and associated gene expression profiles of VEGF-C with long-term outcome remain to be defined. However, several effects of VEGF-C on the treatment and gene expression profiles were investigated by using microarray analysis in 525 adult and 100 pediatric AML patients. The results showed that increased VEGF-C predicted adverse long-term prognosis, which provided additional information to well-known factors (91).

VEGF/VEGFR in chronic myeloid leukemia or chronic lymphocytic leukemia. Aguayo et al (36) evaluated the blood vessels in 145 bone marrow biopsies and the levels of VEGF, bFGF, TNF- $\alpha$, TGF- $\alpha$ and HGF in 417 plasma samples. Except for CLL, vascularity was significantly higher in all leukemias compared with the control bone marrows. The highest number of blood vessels and the largest vascular area were discovered in CML. At the same time the highest levels of VEGF in plasma were detected in CML, while the highest levels of bFGF were in CLL. The level of HGF was highest in chronic myelomonocytic leukemia (CMML). Molica et al (92) evaluated the levels of VEGF in serum in B-CLL, and the results indicated that increased serum levels of VEGF may be considered in predicting the risk of disease progression. Ferrajoli et al (93) discovered VEGFR-2 was a high-affinity VEGF receptor that plays a role in de novo blood vessel formation and hematopoietic cell development. Cellular VEGFR-2 levels may serve as a prognostic factor in CLL. All evidence supports that VEGF is a critical microenvironmental factor, and VEGF inhibition may be a promising new therapeutic approach in CLL. For example, vatalanib and pazopanib seem to be effective and safe candidates. More evaluation will be required to confirm these results.

\section{Antiangiogenic therapy in leukemias}

Antiangiogenic therapy in animal models with leukemias. According to the relationship between VEGF/VEGFR and leukemias, antiangiogenic therapy was used to treat leukemias in animal models. Dias et al (94) reported, using an in vivo model of human leukemia, that blocking angiogenesis induced by the interaction of leukemia-derived VEGF with murine VEGFR-2 delays leukemic growth. Although it was not sufficient for its eradication from inoculated mice, these results demonstrated that targeting VEGF-induced angiogenesis may be at least partially effective in delaying the progression of leukemia. Interestingly, long-term remission was achieved only when mice were treated with neutralizing monoclonal antibody $(\mathrm{mAb})$ against murine and human VEGFR-2, blocking the paracrine and autocrine VEGF-VEGFR-2 signaling pathways. On the other hand, mAbs against murine or human VEGFR-1 had no effect towards improving the survival of the leukemic mice, suggesting that the VEGF/VEGFR-2 pathway is more important for the proliferation and engraftment of acute leukemias in vivo. However, it is also possible that several leukemias may depend on VEGFR-1 signaling (95). In order to further examine the role of VEGF in AML progression in vivo, a number of researchers established two mouse models. In a murine chloroma model, the delivery of VEGF using microencapsulation technology resulted in enhanced tumor growth and vascularization, whereas treatment with a VEGF antagonist soluble NRP-1 (sNRP-1) inhibited tumor angiogenesis and growth. In a systemic leukemia model, the survival of mice injected with adenovirus (Ad) encoding for Fc-sNRP-1 was significantly prolonged as compared with mice injected with Ad-LacZ. Further analysis showed a reduction in circulating leukemic cells and infiltration of liver and spleen as well as bone marrow neovascularization and cellularity (96). Liu et al (97) demonstrated that adenoviral gene therapy with antiangiogenic fragments of thrombospondin-1 inhibited leukemic xenograft growth in mice, which addressed the possibility that reduced production of angiogenesis inhibitors by leukemic cells may trigger the onset of the neovascularization process, by shifting the local (bone marrow) angiogenesis balance.

In contrast to the positive roles in leukemia disease, emerging evidence from genetically modified animal models suggests that elevated levels of VEGF, or a proangiogenic phenotype, may impede, rather than promote, early tumor development and progression (98). Researchers reported a tumor inhibitory role for VEGF by demonstrating that a 
Table I. Summary of clinical trials and approved antiangiogenic therapies in patients with leukemia.

\begin{tabular}{|c|c|c|c|c|c|c|c|}
\hline \multirow[b]{2}{*}{ Patients } & \multicolumn{2}{|c|}{ Anti-VEGF strategies } & \multicolumn{3}{|c|}{ RTK inhibitors } & \multicolumn{2}{|c|}{ Immunomodulators } \\
\hline & Target & Bevacizumab & Target & Vatalanib & Cediranib & Thalidomide & Lenalidomide \\
\hline AML & VEGF-A & + & VEGFR1-3 & + & + & + & + \\
\hline CML & VEGF-A & + & VEGFR1-3 & + & l & / & l \\
\hline CLL & VEGF-A & + & 1 & + & + & + & + \\
\hline
\end{tabular}

+ , There was response to treatment; /, there was no response to treatment. VEGF, vascular endothelial growth factor; VEGFR, VEGF receptor; AML, acute myeloid leukemia; CML, chronic myeloid leukemia; CLL, chronic lymphocytic leukemia.

2-fold overexpression of systemic levels of VEGF in mice heterozygous for a VEGF 'hypermorphic' allele decelerated tumorigenesis in a retroviral-induced, spontaneous murine leukemia model (99). Alterations in the innate immune function, specifically enhanced natural killer cell activity, and increased hematopoietic progenitor cell survival were identified as acquired phenotypes that strongly correlated with and were likely responsible for this leukemic inhibition (99). Accordingly, Ebos et al (100) recently showed that blockade of the VEGF pathway, before tumor induction, led to a more aggressive metastasis and shortened survival. Therefore, further experimental and clinical studies are required to clarify the controversy surrounding the dichotomous roles of VEGF in tumor angiogenesis.

Antiangiogenic therapy in patients with leukemias. The first antiangiogenic agent to be approved was bevacizumab, a humanized anti-VEGF monoclonal antibody. Administration of bevacizumab, in combination with cytotoxic chemotherapy, confers benefits to patients with several types of solid cancers (101-103). Additionally, two small-molecule inhibitors targeting VEGFR and other kinases, sorafenib and sunitinib, have been approved for treating renal cells- and hepatocellular carcinoma $(104,105)$. These are currently under investigation for patients with relapsed and refractory acute leukemia in combination with standard chemotherapy (106). The major classes of antiangiogenic therapy include direct anti-VEGF acting molecules (anti-VEGF antibodies, VEGF-antisense nucleotides), immunomodulatory drugs (IMIDs) with antiangiogenic properties, receptor tyrosine kinase inhibitors that target VEGFR signaling as well as receptors of other (proangiogenic) factors, the anti-endothelial approach of metronomic therapy, and other new compounds targeting signaling downstream to proangiogenic growth factors, such as mTOR inhibitors, histone deacetylase (HDAC) inhibitors and proteasome inhibitors. Moreover, angiogenesis appears to be targeted even by conventional chemotherapy in different leukemias (61) (Table I). For example, bevacizumab is a humanized murine anti-human VEGF monoclonal IgG1 antibody that blocks the binding of human VEGF to its receptors VEGFR-1 and -2 (107). It was administered after chemotherapy to 48 adults with refractory or relapsed AML. The overall response was 23 of $48(48 \%)$, with complete response (CR) in $16(33 \%)$. MVD decreased in the bone marrow after bevacizumab administration. Currently, bevacizumab is being evaluated as a treatment option for newly diagnosed AML patients in combination with cytarabine and idarubicin in a phase II study. Thalidomide is administered as an antineoplastic agent after the demonstration of its antiangiogenic activity (108). The newer IMIDs lenalidomide, a synthetic compound derived by modifying the chemical structure of thalidomide, was observed to have 2-3 times more potent antiangiogenic activity than thalidomide in various in vivo assays (109) and the antiangiogenic activity has been demonstrated to be independent of their immunomodulatory effects (110). In a phase II study by Thomas et al (111), thalidomide was analyzed in 16 patients with relapsed or refractory AML; one patient (6\%) achieved CR lasting for 36 months. There was no correlation between the reduction in angiogenesis marker levels and responses. In a phase I/II trial by Steins et al (112) in 20 AML patients, a partial response was observed in four patients. In parallel, MVD significantly decreased in five patients during treatment with thalidomide. In a study by Barr et al (113), thalidomide was examined in combination with fludarabine, carboplatin, and topotecan in 42 patients with poor AML prognosis and 10 of 42 (24\%) patients achieved a CR. Small tyrosine kinase inhibitors that target VEGFR are a further important class of antiangiogenic drugs. For example, vatalanib is an oral angiogenesis inhibitor that offers a novel approach to inhibiting tumor growth (114) by interfering with the ATP binding sites of VEGFR . Ongoing studies are now focusing on evaluating the efficacy of vatalanib in combination with imatinib in a phase I/II trial for patients with AML, PMF and blast phase of CML, or in combination with cytosine arabinoside and daunorubicin in patients with AML (115). Cediranib (AZD2171, Recentin) is a potent inhibitor of both VEGFR-1 and -2 (116). In a phase I study with cediranib in 35 AML patients, six patients experienced an objective response. There was a correlation between cediranib exposure and plasma VEGF levels (117). A combination therapy of thalidomide and 5-azacytidine, a hypomethylating drug, was assessed in 40 patients with AML (118). Hematological improvement was observed in 15 of 36 patients (42\%), stable disease was observed in 5 of 36 patients (14\%), 10 of 36 patients (28\%) had disease progression and 6 had CR.

\section{Conclusion}

This investigation demonstrates that angiogenesis has important biological and prognostic implications in hematolymphoid malignancies. The autocrine regulators of angiogenesis are essential to the development of diseases. VEGF/VEGFR as key 
regulators of neoangiogenesis and vasculogenesis have been widely studied. Although the mechanism of the high expression of VEGF in leukemia cells has not yet been identified, we are certain that VEGF/VEGFR interactions may stimulate proliferation, migration and survival of leukemia/lymphoma cells by autocrine and paracrine loops. In clinical studies, the elevated level of VEGF may contribute to the adverse outcome by promoting leukemic cell growth, survival, migration and reduce the sensitivity of cells to therapeutic agent-induced apoptosis. Therefore, the interference of VEGF/VEGFRrelated pathway being an ideal candidate in treating leukemia may induce antiangiogenesis and inhibit growth of leukemia cells. All clinical trials causing VEGFR-2 gene polymorphism relates to cytogenetic response (treatment failure following imatinib therapy for CML) and the VEGF genotype relates to the progression of advanced disease. Apart from anti-VEGF molecules, other antiangiogenic therapies include IMIDs, receptor tyrosine kinase inhibitors, anti-endothelial approach to metronomic therapy, mTOR inhibitors, HDAC inhibitors and proteasome inhibitors. A better understanding of the role of VEGF in leukemia and additional trials combining antiangiogenic therapies will provide a greater insight to the mechanisms required for treatment.

\section{Acknowledgements}

This study was supported by the Key Foundation of Shandong Science Project (2007GG2002023), The Shandong 1020 Project (2008-2), The Natural Science Foundation of Shandong (Y2008C165) and the Natural Science Foundation of China (30810444).

\section{References}

1. Kerbel RS: Tumor angiogenesis. N Engl J Med 358: 2039-2049, 2008.

2. Ellis LM and Hicklin DJ: VEGF-targeted therapy: mechanisms of anti-tumour activity. Nat Rev Cancer 8: 579-591, 2008.

3. Medinger M, Fischer N and Tzankov A: Vascular endothelial growth factor-related pathways in hemato-lymphoid malignancies. J Oncol 2010: 729725, 2010. doi: 10.1155/2010/729725.

4. Aguayo A, Kantarjian HM, Estey EH, et al: Plasma vascular endothelial growth factor levels have prognostic significance in patients with acute myeloid leukemia but not in patients with myelodysplastic syndromes. Cancer 95: 1923-1930, 2002.

5. Zhu Z, Hattori K, Zhang H, et al: Inhibition of human leukemia in an animal model with human antibodies directed against vascular endothelial growth factor receptor 2. Correlation between antibody affinity and biological activity. Leukemia 17: 604-611, 2003.

6. Benouchan $\mathrm{M}$ and Colombo BM: Anti-angiogenic strategies for cancer therapy. Int J Oncol 27: 563-571, 2005.

7. Ferrara $\mathrm{N}$ and Henzel WJ: Pituitary follicular cell secrete a novel heparin-binding growth factor specific for vascular endothelial cell. Biochem Biophys Res Commun 161: 851, 1989.

8. Ferrara N, Gerber HP and LeCouter J: The biology of VEGF and its receptors. Nat Med 9: 669-676, 2003.

9. Cao Y: Positive and negative modulation of angiogenesis by VEGFR1 ligands. Sci Signal 2: re1, 2009.

10. Zhang L, Zhou F, Han W, et al: VEGFR-3 ligand-binding and kinase activity are required for lymphangiogenesis but not for angiogenesis. Cell Res 20: 1319-1331, 2010.

11. Wu Y, Hooper AT, Zhong Z, et al: The vascular endothelial growth factor receptor (VEGFR-1) supports growth and survival of human breast carcinoma. Int J Cancer 119: 1519-1529, 2006

12. Autiero M, Luttun A, Tjwa M and Carmeliet P: Placental growth factor and its receptor, vascular endothelial growth factor receptor-1: novel targets for stimulation of ischemic tissue revascularization and inhibition of angiogenic and inflammatory disorders. J Thromb Haemost 1: 1356-1370, 2003.
13. Holmes K, Roberts OL, Thomas AM and Cross MJ: Vascular endothelial growth factor receptor-2: structure, function, intracellular signalling and therapeutic inhibition. Cell Signal 19: 2003-2012, 2007.

14. Brekken RA, Overholser JP, Stastny VA, Waltenberger J, Minna JD and Thorpe PE: Selective inhibition of vascular endothelial growth factor (VEGF) receptor 2 (KDR/Flk-1) activity by a monoclonal anti-VEGF antibody blocks tumor growth in mice. Cancer Res 60: 5117-5124, 2000

15. Katoh O, Tauchi H, Kawaishi K, Kimura A and Satow Y: Expression of the vascular endothelial growth factor (VEGF) receptor gene, KDR, in hematopoietic cells and inhibitory effect of VEGF on apoptotic cell death caused by ionizing radiation. Cancer Res 55: 5687-5692,1995.

16. Cursiefen C, Chen L, Borges LP, et al: VEGF-A stimulates lymphangiogenesis and hemangiogenesis in inflammatory neovascularization via macrophage recruitment. J Clin Invest 113: 1040-1050, 2004.

17. Adams J, Carder PJ, Downey S, et al: Vascular endothelial growth factor (VEGF) in breast cancer: comparison of plasma, serum and tissue VEGF and microvessel density and effects of tamoxifen. Cancer Res 60: 2898-2905, 2000.

18. Kay NE, Bone ND, Tschumper RC, et al: B-CLL cells are capable of synthesis and secretion of both pro- and anti-angiogenic molecules. Leukemia 16: 911-919, 2002.

19. Rafii S, Lyden D, Benezra R, Hattori K and Heissig B: Vascular and haematopoietic stem cells: novel targets for anti-angiogenesis therapy? Nat Rev Cancer 2: 826-835, 2002.

20. Shweiki D, Itin A, Soffer D and Keshet E: Vascular endothelial growth factor induced by hypoxia may mediate hypoxia-initiated angiogenesis. Nature 359: 843-845, 1992.

21. Dor Y, Porat R and Keshet E: Vascular endothelial growth factor and vascular adjustments to perturbations in oxygen homeostasis. Am J Physiol Cell Physiol 280: C1367-C1374, 2001.

22. Kaelin WG Jr: The von Hippel-Lindau tumor suppressor protein and clear cell renal carcinoma. Clin Cancer Res 13: 680s-682s, 2007.

23. Cong XL, Li B, Yang RC, Feng SZ, Chen SJ and Han ZC: Enhanced growth suppression of Philadephial leukemia cells by targeting bcr3/abl2 and VEGF through antisense strategy. Leukemia 19: 1517-1524, 2005.

24. Ghosh AK, Shanafelt TD, Cimmino A, et al: Aberrant regulation of pVHL levels by microRNA promotes the HIF/VEGF axis in CLL B cells. Blood 113: 5568-5574, 2009.

25. Withey JM, Marley SB, Kaeda J, Harvey AJ, Crompton MR and Gordon MY: Targeting primary human leukemia cells with RNA interference: Bcr-Abl targeting inhibits myeloid progenitor selfrenewal in chronic myeloid leukaemia cells. Br J Haematol 129: 377-380, 2005.

26. Schmittgen TD and Livak KJ: Analyzing real-time PCR data by the comparative C(T) method. Nat Protoc 3: 1101-1108, 2008.

27. Ghosh AK, Secreto CR, Knox TR, Ding W, Mukhopadhyay D and Kay NE: Circulating microvesicles in B-cell chronic lymphocytic leukemia can stimulate marrow stromal cells: implications for disease progression. Blood 115: 1755-1764, 2010.

28. Aggarwal BB, Sethi G, Ahn KS, et al: Targeting signaltransducer-and-activator-of-transcription-3 for prevention and therapy of cancer:modern target but ancient solution. Ann NY Acad Sci 1091: 151-169, 2006.

29. Eriksen KW, Kaltoft K, Mikkelsen G, et al: Constitutive STAT3activation in Sezary syndrome: tyrphostin AG490 inhibits STAT3-activation, interleukin-2 receptor expression and growth of leukemic Sezary cells. Leukemia 15: 787-793, 2001.

30. Nielsen M,Nissen MH, Gerwien J, et al: Spontaneous interleukin-5 production in cutaneous T-cell lymphoma lines is mediated by constitutively activated Stat3. Blood 99: 973-977, 2002.

31. Minet E, Michel G, Mottet D, et al: c-JUN gene induction and AP-1 activity is regulated by a JNK-dependent pathway in hypoxic HepG2 cells. Exp Cell Res 265: 114-124, 2001.

32. Pages G, Berra E, Milanini J, Levy AP and Pouyssegur J: Stressactivated protein kinases (JNK and p38/HOG) are essential for vascular endothelial growth factor mRNA stability. J Biol Chem 275: 26484-26491, 2000.

33. Krejsgaard T, Vetter-Kauczok CS, Woetmann A, et al: Jak3- and JNK-dependent vascular endothelial growth factor expression in cutaneous T-cell lymphoma. Leukemia 20: 1759-1766, 2006.

34. Kumar SA, Hu X, Brown M, et al: Lysophosphatidic acid receptor expression in chronic lymphocytic leukemia leads to cell survival mediated though vascular endothelial growth factor expression. Leuk Lymphoma 50: 2038-2048, 2009. 
35. Hu X, Mendoza FJ, Sun J, Banerji V, Johnston JB and Gibson SB: Lysophosphatidic acid (LPA) induces the expression of VEGF leading to protection against apoptosis in B-cell derived malignancies. Cell Signal 20: 1198-1208, 2008.

36. Aguayo A, Kantarjian H, Manshouri T, et al: Angiogenesis in acute and chronic leukemias and myelodysplastic syndromes Blood 96: 2240-2245, 2000.

37. Bellamy WT, Richter L, Sirjani D, et al: Vascular endothelial cell growth factor is an autocrine promoter of abnormal localized immature myeloid precursors and leukemia progenitor formation in myelodysplastic syndromes. Blood 97: 1427-1434, 2001.

38. Braun T, Carvalho G, Fabre C, Grosjean J, Fenaux P and Kroemer G: Targeting NF-kappaB in hematologic malignancies. Cell Death Differ 13: 748-758, 2006.

39. Zhao T, Yasunaga J, Satou Y, et al: Human T-cell leukemia virus type $1 \mathrm{bZIP}$ factor selectively suppresses the classical pathway of NF-kappaB. Blood 113: 2755-2764, 2009.

40. Sujobert P, Bardet V, Cornillet-Lefebvre P, et al: Essential role for the p110delta isoform in phosphoinositide 3-kinase activation and cell proliferation in acute myeloid leukemia. Blood 106 1063-1066, 2005.

41. Ramos AM, Fernandez C, Amran D, Sancho P, de Blas E and Aller P: Pharmacologic inhibitors of PI3K/Akt potentiate the apoptotic action of the antileukemic drug arsenic trioxide via glutathione depletion and increased peroxide accumulation in myeloid leukemia cells. Blood 105: 4013-4020, 2005.

42. Shi Q, Le X, Abbruzzese JL, et al: Constitutive Sp1 activity is essential for differential constitutive expression of vascular endothelial growth factor in human pancreatic adenocarcinoma. Cancer Res 61: 4143-4154, 2001.

43. Hsu TC, Young MR, Cmarik J and Colburn NH: Activator protein 1 (AP-1) and nuclear factor kappaB (NF-kappa B)-dependent transcriptional events in carcinogenesis. Free Radic Biol Med 28: $1338-1348,2000$

44. Angel P and Karin M: The role of Jun, Fos and the AP-1 complex in cell proliferation and transformation. Biochim Biophys Acta 1072: 129-157, 1991.

45. Pollmann C, Huang X, Mall J, Bech-Otschir D, Naumann M and Dubiel W: The constitutive photomorphogenesis 9 signalosome directs vascular endothelial growth factor production in tumor cells. Cancer Res 61: 8416-8421, 2001.

46. Poulaki V, Mitsiades CS, McMullan C, et al: Regulation of vascular endothelial growth factor expression by insulin-like growth factor I in thyroid carcinomas. J Clin Endocrinol Metab 88: 5392-5398, 2003.

47. Chuang $\mathrm{CH}$, Huang $\mathrm{CS}$ and $\mathrm{Hu}$ ML: Vitamin $\mathrm{E}$ and rutin synergistically inhibit expression of vascular endothelial growth factor through down-regulation of binding activity of activator protein-1 in human promyelocytic leukemia (HL-60) cells. Chem Biol Interact 183: 434-441, 2010.

48. Rho SB, Choi K, Park K and Lee JH: Inhibition of angiogenesis by the BTB domain of promyelocytic leukemia zinc finger protein. Cancer Lett 294: 49-56, 2010.

49. Konopka JB, Watanabe SM and Witte ON: An alteration of the human c-abl protein in K562 leukemia cells unmasks associated tyrosine kinase activity. Cell 37: 1035-1042, 1984.

50. Pendergast AM, Quilliam LA, Cripe LD, et al: BCR-ABLinduced oncogenesis is mediated by direct interaction with the SH2 domain of the GRB-2 adaptor protein. Cell 75: 175-185, 1993.

51. Carlesso N, Frank DA and Griffin JD: Tyrosyl phosphorylation and DNA binding activity of signal transducers and activators of transcription (STAT) proteins in hematopoietic cell lines transformed by Bcr/Abl. J Exp Med 183: 811-820, 1996.

52. Sillaber C, Gesbert F, Frank DA, Sattler M and Griffin JD: STAT5 activation contributes to growth and viability in $\mathrm{Bcr} /$ Abl-transformed cells. Blood 95: 2118-2125, 2000.

53. Nieborowska-Skorska M, Wasik MA, Slupianek A, Salomoni P, Kitamura T, Calabretta B and Skorski T: Signal transducer and activator of transcription (STAT) 5 activation by BCR/ABL is dependent on intact $\mathrm{Src}$ homology (SH)3 and $\mathrm{SH} 2$ domains of $\mathrm{BCR} / \mathrm{ABL}$ and is required for leukemogenesis. J Exp Med 189 1229-1242, 1999.

54. Skorski T, Bellacosa A, Nieborowska-Skorska M, Majewski M, Martinez R, Choi JK, et al: Transformation of hematopoietic cells by $\mathrm{BCR} / \mathrm{ABL}$ requires activation of a PI-3k/Akt-dependent pathway. EMBO J 16: 6151-6161, 1997.

55. Hwan D, Kim, Xu W, et al: Lipton genetic variants in the candidate genes of the apoptosis pathway and susceptibility to chronic myeloid leukemia. Blood 113: 2517-2525, 2009.
56. Li L, Zhang R, Fang ZY, Chen JN and Zhu ZL: Suppression of vascular endothelial growth factor (VEGF) expression by targeting the Bcr-Abl oncogene and protein tyrosine kinase activity in Bcr-Abl-positive leukaemia cells. J Int Med Res 37: 426-437, 2009.

57. Böhm A, Aichberger KJ, Mayerhofer M, et al: Targeting of mTOR is associated with decreased growth and decreased VEGF expression in acute myeloid leukaemia cells. Eur J Clin Invest 39: 395-405, 2009

58. Venables JP: Unbalanced alternative splicing and its significance in cancer. Bioessays 28: 378-386, 2006.

59. Podar K, Tai YT and Davies FE: Vascular endothelial growth factor triggers signaling cascades mediating multiple myeloma cell growth and migration. Blood 98: 428-435, 2001.

60. Hussong JW, Rodgers GM and Shami PJ: Evidence of increased angiogenesis in patients with acute myeloid leukemia. Blood 95: 390-313, 2000

61. Padró T, Ruiz S, Bieker R, Bürger H, Steins M, Kienast J, et al: Increased angiogenesis in the bone marrow of patients with acute myeloid leukemia. Blood 95: 2637-2644, 2000.

62. Abrams ST, Lakum T, Lin K, et al: B-cell receptor signaling in chronic lymphocytic leukemia cells is regulated by overexpressed active protein kinase CßII. Blood 109: 1193-1201, 2007.

63. Gora-Tybor J, Blonski JZ and Robak T: Circulating vascular endothelial growth factor (VEGF) and its soluble receptors in patients with chronic lymphocytic leukemia. Eur Cytokine Netw 16: 41-46, 2005.

64. Abrams ST, Brown BR, Zuzel M and Slupsky JR: Vascular endothelial growth factor stimulates protein kinase C $\beta I I$ expression in chronic lymphocytic leukaemia cells. Blood 115: 4447-4454, 2010.

65. Ugarte-Berzal E, Redondo-Muñoz J, Eroles P, et al: VEGF/ VEGFR2 interaction down-regulates matrix metalloproteinase-9 via STAT1 activation and inhibits B chronic lymphocytic leukemia cell migration. Blood 115: 846-849, 2010.

66. Hata AN and Breyer RM: Pharmacology and signaling of prostaglandin receptors: multiple roles in inflammation and immune modulation. Pharmacol Ther 103: 147-166, 2004

67. Wu G,Luo J, Rana JS, Laham R, Sellke FW and Li J: Involvement of COX-2 in VEGF-induced angiogenesis via P38 and JNK pathways in vascular endothelial cells. Cardiovasc 69: 512-519, 2006.

68. Su JL, Shih JY, Yen ML, et al: Cyclooxygenase-2 induces EP1and HER-2/Neu-dependent vascular endothelial growth factor-C up-regulation: a novel mechanism of lymphangiogenesis in lung adenocarcinoma. Cancer Res 64: 554-564, 2004.

69. Véronèse L, Tournilhac O, Verrelle P, et al: Low MCL-1 mRNA expression correlates with prolonged survival in B-cell chronic lymphocytic leukemia. Leukemia 22: 1291-1293, 2008.

70. Pepper C, Lin TT, Pratt G, et al: Mcl-1 expression has in vitro and in vivo significance in chronic lymphocytic leukemia and is associated with other poor prognostic markers. Blood 112: 3807-3817, 2008.

71. Véronèse $\mathrm{L}$, Tournilhac $\mathrm{O}$, Verrelle $\mathrm{P}$, et al: Strong correlation between VEGF and MCL-1 mRNA expression levels in B-cell chronic lymphocytic leukemia. Leuk Res 33: 1623-1626, 2009.

72. Perez-Atayde AR, Sallan SE, Tedrow U, Connors S, Allred E and Folkman J: Spectrum of tumor angiogenesis in the bone marrow of children with acute lymphoblastic leukemia. Am J Pathol 150: 815-821, 1997.

73. Yetgin S, Yenicesu I, Etin MC and Tuncer M: Clinical importance of serum vascular endothelial and basic fibroblast growth factors in children with acute lymphoblastic leukemia. Leuk Lymphoma 42: 83-88, 2001.

74. Dong X, Han ZC and Yang R: Angiogenesis and antiangiogenic therapy in hematologic malignancies. Crit Rev Oncol Hematol 62: 105-118, 2007.

75. Frater JL, Kay NE, Goolsby CL, Crawford SE, Dewald GW and Peterson LC: Dysregulated angiogenesis in B-chronic lymphocytic leukemia: morphologic, immunohistochemical, and flow cytometric evidence. Diagn Pathol 3: 16, 2008.

76. Norén-Nyström U, Heyman M, Frisk P, et al: Vascular density in childhood acute lymphoblastic leukaemia correlates to biological factors and outcome. Br J Haematol 146: 521-530, 2009.

77. Chen H, Treweeke AT, West DC, Till KJ, Cawley JC, Zuzel M and Toh $\mathrm{CH}$ : In vitro and in vivo production of vascular endothelial growth factor by chronic lymphocytic leukemia cells. Blood 96: 3181-3187, 2000.

78. Folkman J: Fundamental concepts of the angiogenic process. Curr Mol Med 3: 643-651, 2003.

79. Verstovsek S, Lunin S, Kantarjian H, et al: Clinical relevance of VEGF receptors 1 and 2 in patients with chronic myelogenous leukemia. Leuk Res 27: 661-669, 2003. 
80. Hayashibara T, Yamada Y, Miyanishi T, et al: Vascular endothelial growth factor and cellular chemotaxis: a possible autocrine pathway in adult T-cell leukemia cell invasion. Clin Cancer Res 7: 2719-2726, 2001.

81. Demacq C, Vasconcellos VB, Izidoro-Toledo TC, et al: Vascular endothelial growth factor (VEGF) and endothelial nitric oxide synthase (NOS3) polymorphisms are associated with high relapse risk in childhood acute lymphoblastic leukemia (ALL). Clin Chim Acta 411: 1335-1405, 2010.

82. Fragoso R, Elias AP and Dias S: Autocrine VEGF loops, signaling pathways, and acute leukemia regulation. Leuk Lymphoma 48: 481-488, 2007.

83. Bellamy WT: Expression of vascular endothelial growth factor and its receptors in multiple myeloma and other hematopoietic malignancies. Semin Oncol 28: 551-559, 2001.

84. Bairey O, Boycov O, Kaganovsky E, Zimra Y, Shaklai M and Rabizadeh E: All three receptors for vascular endothelial growth factor (VEGF) are expressed on B-chronic lymphocytic leukemia (CLL) cells. Leuk Res 28: 243-248, 2004.

85. de Bont ES, Neefjes VM, Rosati S, Vellenga E and Kamps WA: New vessel formation and aberrant VEGF/VEGFR signaling in acute leukemia: does it matter? Leuk Lymphoma 43: 1901-1909, 2002.

86. Aguayo A, Estey E, Kantarjian H, et al: Cellular vascular endothelial growth factor is a predictor of outcome in patients with acute myeloid leukemia. Blood 94: 3717-3721, 1999.

87. Fu J, Fu J, Chen X, Zhang Y, Gu H and Bai Y: CD147 and VEGF co-expression predicts prognosis in patients with acute myeloid leukemia. Jpn J Clin Oncol 40: 1046-1052, 2010.

88. Fiedler W, Graeven U, Ergün S, et al: Vascular endothelial growth factor, a possible paracrine growth factor in human acute myeloid leukemia. Blood 89: 1870-1875, 1997.

89. Koistinen P, Siitonen T, Mäntymaa P, et al: Regulation of the acute myeloid leukemia cell line OCI/AML-2 by endothelial nitric oxide synthase under the control of a vascular endothelial growth factor signaling system. Leukemia 15: 1433-1441, 2001.

90. Fielder W, Graeven U, Ergün S, et al: Expression of FLT4 and its ligand VEGF-C in acute myeloid leukemia. Leukemia 11: 1234-1237, 1997.

91. de Jonge HJ, Valk PJ, Veeger NJ, et al: High VEGFC expression is associated with unique gene expression profiles and predicts adverse prognosis in pediatric and adult acute myeloid leukemia. Blood 116: 1747-1754, 2010.

92. Molica S, Vitelli G, Levato D, Gandolfo GM and Liso V: Increased serum levels of vascular endothelial growth factor predict risk of progression in early B-cell chronic lymphocytic leukaemia. Br J Haematol 107: 605-610, 1999.

93. Ferrajoli A, Manshouri T, Estrov Z, et al: High levels of vascular endothelial growth factor receptor-2 correlate with shortened survival in chronic lymphocytic leukemia. Clin Cancer Res 7: 795-799, 2001.

94. Dias S, Hattori K, Zhu Z, et al: Autocrine stimulation of VEGFR-2 activates human leukemic cell growth and migration. J Clin Invest 106: 511-521, 2000.

95. Dias S, Hattori K, Heissig B, et al: Inhibition of both paracrine and autocrine VEGF/VEGFR-2 signaling pathways is essential to induce long-term remission of xenotransplanted human leukemias. Proc Natl Acad Sci USA 98: 10857-10862, 2001.

96. Schuch G, Machluf M, Bartsch G Jr, et al: In vivo administration of vascular endothelial growth factor (VEGF) and its antagonist, soluble neuropilin-1, predicts a role of VEGF in the progression of acute myeloid leukemia in vivo. Blood 100: 4622-4628, 2002.

97. Liu P, Wang Y, Li YH, et al: Adenovirus-mediated gene therapy with an antiangiogenic fragment of thrombospondin-1 inhibits human leukemia xenograft growth in nude mice. Leuk Res 27: 701-708, 2003

98. Vecchiarelli-Federico LM, Cervi D, Haeri M, Li Y, Nagy A and Ben-David Y: Vascular endothelial growth factor - a positive and negative regulator of tumor growth. Cancer Res 70: 863-867, 2010.
99. Cervi D, Shaked Y, Haeri M, et al: Enhanced natural-killer cell and erythropoietic activities in VEGF-A-overexpressing mice delay F-MuLV-induced erythroleukemia. Blood 109: 2139-2146, 2007.

100. Ebos JM,Lee CR, Cruz-Munoz W, Bjarnason GA, Christensen JG and Kerbel RS: Accelerated metastasis after short-term treatment with a potent inhibitor of tumor angiogenesis. Cancer Cell 15: 232-239, 2009

101. Hurwitz H, Fehrenbacher L, Novotny W, et al: Bevacizumab plus irinotecan, fluorouracil and leucovorin for metastatic colorectal cancer. N Engl J Med 350: 2335-2342, 2004.

102. Sandler A, Gray R, Perry MC, et al: Paclitaxel-carboplatin alone or with bevacizumab for non-small-cell lung cancer. N Engl J Med 355: 2542-2550, 2006.

103. Miller K, Wang M, Gralow J, et al: Paclitaxel plus bevacizumab versus paclitaxel alone for metastatic breast cancer. N Engl J Med 357: 2666-2676, 2007.

104. Escudier B, Eisen T, Stadler WM, et al: Sorafenib in advanced clear-cell renal-cell carcinoma. N Engl J Med 356: 125-134, 2007.

105. Motzer RJ, Hutson TE, Tomczak P, et al: Sunitinib versus interferon alfa in metastatic renal-cell carcinoma. N Engl J Med 356: 115-124, 2007.

106. Karp JE, Gojo I, Pili R, et al: Targeting vascular endothelial growth factor for relapsed and refractory adult acute myelogenous leukemias: therapy with sequential $1-\beta$-d-arabinofuranosylcytosine, mitoxantrone, and bevacizumab. Clin Cancer Res 10: 3577-3585, 2004.

107. Presta LG, Chen H, O'Connor SJ, et al: Humanization of an anti-vascular endothelial growth factor monoclonal antibody for the therapy of solid tumors and other disorders. Cancer Res 57: 4593-4599, 1997.

108. D'Amato RJ, Loughnan MS, Flynn Eand Folkman J: Thalidomide is an inhibitor of angiogenesis. Proc Natl Acad Sci USA 91: 4082-4085, 1994.

109. Teo SK: Properties of thalidomide and its analogues: implications for anticancer therapy. AAPS J 7: E14-E19, 2005.

110. Dredge K, Marriott JB, Macdonald CD, et al: Novel thalidomide analogues display anti-angiogenic activity independently of immunomodulatory effects. Br J Cancer 87: 1166-1172, 2002.

111. Thomas DA, Estey E, Giles FJ, et al: Single agent thalidomide in patients with relapsed or refractory acute myeloid leukaemia. Br J Haematol 123: 436-441, 2003.

112. Steins MB, Padró T, Bieker R, et al: Efficacy and safety of thalidomide in patients with acute myeloid leukemia. Blood 99: 834-839, 2002.

113. Barr P, Fu P, Lazarus H, et al: Antiangiogenic activity of thalidomide in combination with fludarabine, carboplatin and topotecan for high-risk acute myelogenous leukemia. Leuk Lymphoma 48: 1940-1949, 2007.

114. Drevs J, Müller-Driver R, Wittig C, et al: PTK787/ZK 222584, a specific vascular endothelial growth factor-receptor tyrosine kinase inhibitor, affects the anatomy of the tumor vascular bed and the functional vascular properties as detected by dynamic enhanced magnetic resonance imaging. Cancer Res 62: 4015-4022, 2002.

115. Roboz GJ, Giles FJ, List AF, et al: Phase 1 study of PTK787/ ZK 222584, a small molecule tyrosine kinase receptor inhibitor, for the treatment of acute myeloid leukemia and myelodysplastic syndrome. Leukemia 20: 952-957, 2006.

116. Wedge SR, Kendrew J, Hennequin LF, et al: AZD2171: a highly potent, orally bioavailable, vascular endothelial growth factor receptor-2 tyrosine kinase inhibitor for the treatment of cancer. Cancer Res 65: 4389-4400, 2005.

117. Fiedler W, Mesters R, Heuser M, et al: An open-label, phase I study of cediranib (RECENTIN) in patients with acute myeloid leukemia. Leuk Res 34: 196-202, 2010.

118. Raza A, Mehdi M, Mumtaz M, Ali F, Lascher S and Galili N: Combination of 5-azacytidine and thalidomide for the treatment of myelodysplastic syndromes and acute myeloid leukemia. Cancer 113: 1596-1604, 2008. 